\title{
Arthroscopic superior capsular reconstruction of the shoulder: a narrative review
}

\author{
Erica Kholinne ${ }^{1,2}$, In-Ho Jeon ${ }^{2} \wedge$ \\ ${ }^{1}$ Faculty of Medicine, Trisakti University, Department of Orthopedic Surgery, St. Carolus Hospital, Jakarta, Indonesia; ${ }^{2}$ Department of Orthopaedic \\ Surgery, University of Ulsan, College of Medicine, Asan Medical Center, Seoul, Korea \\ Contributions: (I) Conception and design: All authors; (II) Administrative support: All authors; (III) Provision of study materials or patients: All \\ authors; (IV) Collection and assembly of data: All authors; (V) Data analysis and interpretation: All authors; (VI) Manuscript writing: All authors; (VII) \\ Final approval of manuscript: All authors. \\ Correspondence to: In-Ho Jeon, MD, PhD. Department of Orthopaedic Surgery, Asan Medical Center, School of Medicine, University of Ulsan, 86 \\ Asanbyeongwon-gil, Songpa-gu, Seoul, Korea. Email: jeonchoi@gmail.com.
}

\begin{abstract}
Irreparable rotator cuff tears (IRCTs) in young and considerably active patients are difficult to treat because it is mostly associated with poor outcome which may lead to a painful and dysfunctional shoulder. Most of the IRCTs are encountered in massive size rotator cuff tears which associated with high failure rate following surgical repair. Thus, the IRCTs was considered challenging for its poor healing rate following repair which may induce the arthritic changes. Since the advent of arthroscopic superior capsular reconstruction (ASCR) of the shoulder in 2013, it has gained its popularity. The procedure has become the most popular option for joint-preserving shoulder surgery for patients with IRCTs. It works by providing a static restraint to the superior humeral head migration to optimize the rotator cuff force couples, hence improving joint kinematics. The acceptance of superior capsular reconstruction has made it rapidly evolving in terms of a wider variety of procedures and broader surgical indications. Despite the enthusiasm and widely acceptance towards the procedure, there are still many queries that exist regarding the best indications, surgical technique particularly graft of choice, the long-term outcome, and the complication and risk of the superior capsular reconstruction (SCR). This narrative review provide the current evidence of SCR in an attempt to provide a state-of-the-art knowledge.
\end{abstract}

Keywords: Irreparable rotator cuff tears (IRCTs); superior capsular reconstruction

Submitted Aug 18, 2020. Accepted for publication Jan 08, 2021.

doi: $10.21037 / \mathrm{atm}-20-5925$

View this article at: http://dx.doi.org/10.21037/atm-20-5925

\section{Introduction}

Irreparable rotator cuff tears (IRCTs) are difficult to treat for it is mostly associated with massive tear size. The increase in rotator cuff tear size and patient age are reported to be associated with the poor outcome and higher failure rate following surgical repair (1). The massive IRCTs are reported to have $79 \%$ retear rate following primary surgical repair (2). Hence, the IRCTs was considered as challenging case due to its rate of poor healing, which is often later associated with arthritic changes (3). In the literature, various joint-preserving surgical options for the treatment of massive irreparable rotator cuff tears (MIRCT) have been reported such as debridement procedures, long head bicep tenotomies (4), tuberoplasties (5), partial repairs (6), and tendon transfers (7). Over the past few years, the biological augmentation of rotator cuff repairs using patch graft (8) with or without

$\wedge$ ORCID: 0000-0002-9289-9193. 
scaffolds has increased in utilization. However, the biological augmentation cannot withstand the test of time because limitations exist in terms of durability and indications. Tendon transfer using latissimus dorsi tendon has also serve as an option for MIRCTs with the benefit of having greater external rotation motion. However unlike the SCR, the indication of latissimus dorsi tendon transfer was limited to the patient without pseudo-paralysis $(9,10)$. Reverse shoulder arthroplasty is a treatment option for IRCTs but there are concerns regarding its longevity, especially in the young population (11). Thus, joint-preserving surgery such as SCR (12) under arthroscopic guidance with fascia lata autograft (13-18) and allografts (19-22) has been advocated for the younger population with IRCTs.

Arthroscopic SCR (ASCR) is probably the most popular topic in shoulder surgery nowadays. This enthusiasm for SCR indicates the difficulty of the problem it is intended to address: an IRCT in the patient that is poorly suited for an alternative procedure. SCR has been first introduced in 2013 by Mihata et al. (23). Theoretically, it works by providing a superior static restraint to the superior migration of the humeral head. The premise of the procedure is to optimize the rotator cuff force couples, thus improving joint kinematics (24). SCR represents a valuable additional tool for the shoulder surgeon and not a universal solution for every challenging rotator cuff tear. Many questions still exist regarding the best indications, surgical technique, the long-term outcome, the complications, and the risk of the procedure. This study reviews the current evidence of SCR in an attempt to provide a state-of-the-art knowledge.

We present the following article in accordance with the Narrative Review reporting checklist (available at http:// dx.doi.org/10.21037/atm-20-5925).

\section{The biomechanics of superior capsule of the shoulder}

The rotator cuff tendon provides a dynamic stability to the glenohumeral joint, which restraints the superior migration of the humeral head when the deltoid muscle is activated. The muscles at the coronal plane (the deltoid and the supraspinatus muscle) and the transverse plane (the infraspinatus, the teres minor, and the subscapularis muscle) work synergistically to provide a balanced force couple. Disruption to the balanced force couple caused by a large rotator cuff tear or MIRCT will result in the proximal migration of the humeral head (25). In addition, this will decrease the efficiency of the biomechanics of the glenohumeral joint because it requires more force to abduct and elevate the arm. When it continuously occurs, this will lead to the deterioration of the shoulder joint function that leads to fixed humeral head migration, further extension of the tear, and eventually the end stage, that is, rotator cuff arthropathy.

In our clinical practice, the choice of SCR has been overlooked because the superior capsule function was ignored in the past (26). Further biomechanics and anatomic studies have reported that the superior capsule transmits force from the rotator cuff muscles and serves as a passive glenohumeral joint stabilizer $(24,27,28)$. In MIRCT, the humeral head migrates proximally at active elevation. Superior capsule reconstruction is performed to restore shoulder joint stability, hence allowing normal joint kinematics and functional outcome (29-32).

\section{Surgical indications}

Medium-term or long-term follow-up studies on the outcome of this relatively new procedure are limited. Most published studies that reported its outcomes are with either without control group or technically driven, which makes it difficult to recognize which patients benefit more from SCR. Thus, the indication for SCR remains indefinite as to which patients are best indicated for this procedure. The surgical indication and contraindication used in our institution are presented in Table 1.

Besides the abovementioned indications and contraindication, patients with pseudoparalysis with associated superior glenohumeral instability are not ideal candidates for reverse shoulder arthroplasty because of young age, and desired activity level may also serve as a good candidate for SCR $(34,35)$. Above all, patients with severe fatty infiltration [Goutalier (36) stage 4] may have less predictable outcomes after rotator cuff repair, even if the tissue is considered repairable. Therefore, these patients may also be candidates for SCR. Adversely, patients with substantial medical comorbidities or poor bone quality (risk of anchor pullout) and those unwilling to comply with postoperative rehabilitation regime are not appropriate candidates for SCR.

\section{Preoperative assessment}

\section{History and physical exam}

The initial workup for SCR candidate patients is similar with any surgical procedure to treat rotator cuff tears. 
Table 1 Surgical indications and contraindications of SCR

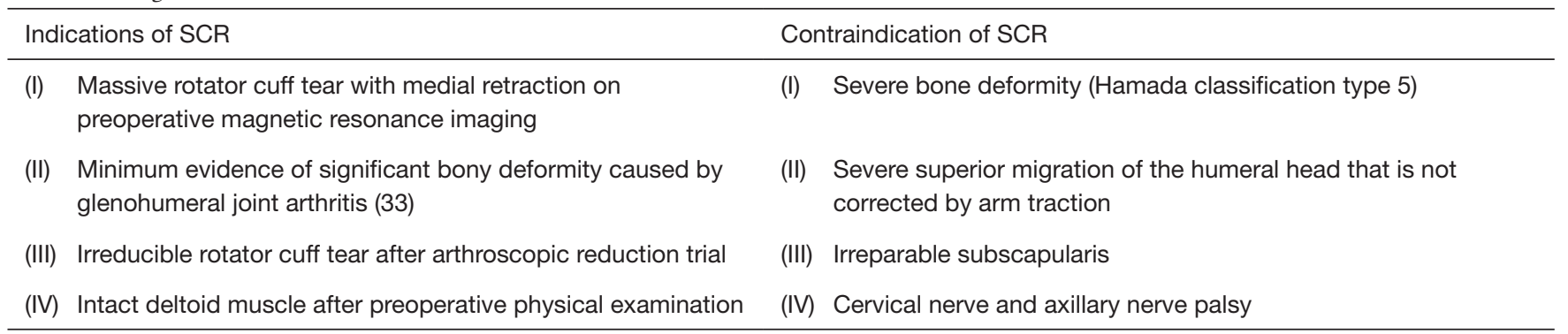
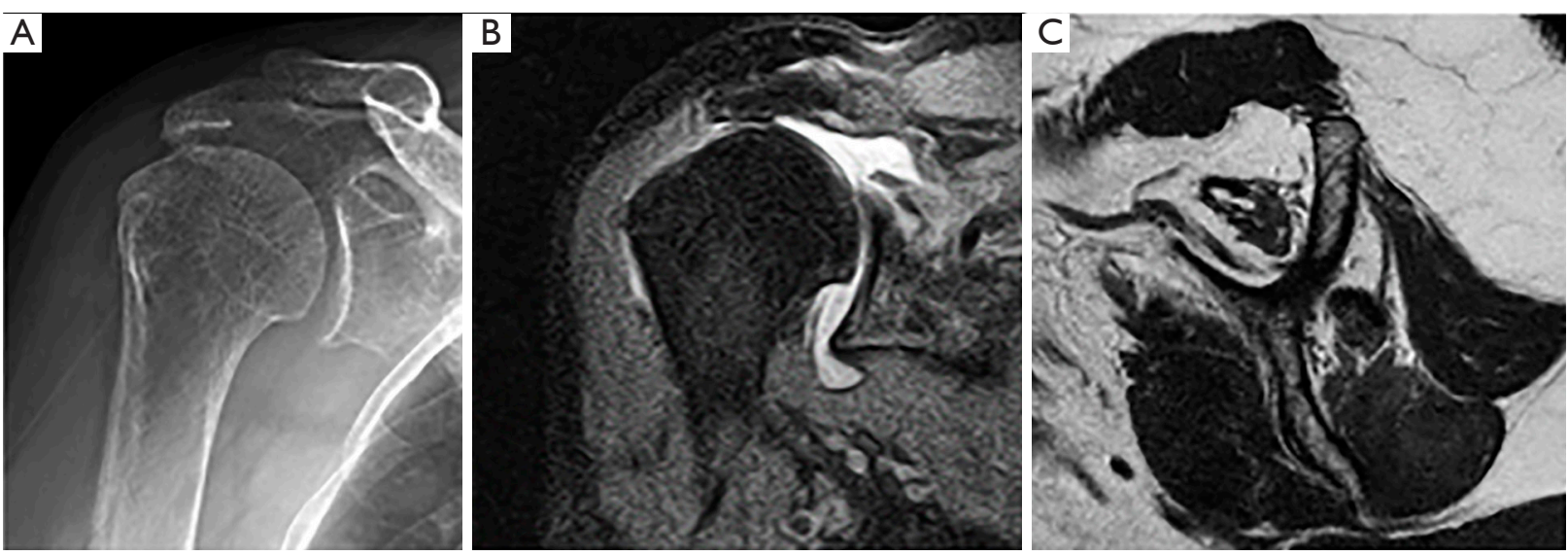

Figure 1 Radiologic assessment showing proximal humeral migration, decreased acromiohumeral distance, and minimal arthritic changes at the glenohumeral joint (A), T2-weighted MRI in coronal plane showing massive rotator cuff tear retracted at the glenoid level (B) and T1weighted MRI in sagittal plane showing stage III fatty infiltration of the rotator cuff muscle (C).

A thorough physical examination for all patients should be performed on both shoulders to assess the status of muscle atrophy. The presence of pseudoparalysis, which is an inability to actively raise the arm above $90^{\circ}$ with a full painless passive motion, should be recorded (37). It is important to note why a patient is having pseudoparalysis, because this is multifactorial, including weakness, severe superior humeral head migration, and significant pain. The cause of pseudoparalysis, together with imaging and intraoperative findings, may have an impact on surgical decision making (38).

Because a concomitant subscapularis repair may be needed at the time of SCR, it is also important to assess the status of subscapularis muscle strength. The repairability of the subscapularis tendon in the presence of a tear is also found to be a prognostic factor of SCR because an irreparable subscapularis tear will have less postoperative muscle strength than those with intact or repairable subscapularis $(39,40)$. All possible sources of pain should be documented, including the palpation of the long head of the biceps tendon and acromioclavicular joint. An assessment into the cervical spine pathology should not be missed.

\section{Imaging assessment}

Preoperative imaging assessment using standard shoulder plain radiographs will provide information regarding the presence of arthritic changes of the shoulder joint, the degree of proximal humeral migration, and the acromiohumeral distance (Figure 1). The acromiohumeral distance is best evaluated on an X-ray taken with the beam tilted $20^{\circ}$ caudally in anteroposterior projection (41). Magnetic resonance imaging (MRI) may provide information regarding the involved tendons, tear size, and fatty infiltration according to Goutallier's index $(36,42)$. A detailed assessment on the fatty infiltration of the rotator cuff muscle should be noted on MRI scan. Expert opinion indicated that rotator cuff tears with severe chronicity with respect to the corresponding 
fatty infiltration "can be arthroscopically repaired" (43). An increased fatty infiltration of the infraspinatus muscle was shown to have a negative effect to the prognosis of SCR (44). However, the status of fatty infiltration of the supraspinatus muscle did not indicate any relationship with the prognosis of SCR. Studies have reported that MRI is not a reliable predictor of rotator cuff repairability $(45,46)$. Nevertheless, it is unclear as to what extent that MRI can predict the success

Table 2 Surgical sequences of SCR
1. Diagnostic arthroscopy
2. Graft harvesting and preparation
3. Acromioplasty
4. Assessment of rotator cuff tear
5. Glenoid site preparation
6. Graft shuttling and fixation at the glenoid side
7. Humeral-site preparation and fixation
8. Remnant bursal coverage or rotator cuff repair

of rotator cuff tear repair.

\section{Surgical technique}

SCR was usually performed with a designated surgical sequence to ease and control the surgical timing (Table 2).

The SCR requires a long learning curve in time and patience. SCR was usually performed for approximately 135-150 minutes with the help of an experienced and dedicated team (first assistant, arthroscopy work; second assistant, graft work) as can be seen in the surgical time frame. The first assistant will assist with the arthroscopic work while the second assistant will work on the graft preparation. According to our experience, the surgical time checklist provided an orchestrated work from all team members to control the surgical time (Table 3).

\section{Diagnostic arthroscopy}

For SCR, a direct lateral portal was used as the main viewing portal in a standard beach chair position. A

Table 3 SCR surgical time frame

\begin{tabular}{|c|c|c|}
\hline Allocated time (minutes) & \multicolumn{2}{|c|}{ ASCR with fascia lata autograft (mesh) } \\
\hline $10-15$ & Diagnostic arthroscopy and rotator cuff tear assessment & \\
\hline 12 & Graft harvesting & Graft cleaning \\
\hline 10 & Acromioplasty & Graft sizing and suturing \\
\hline 5 & Glenoid anchoring & \\
\hline 6 & Glenoid anchors limbs' suturing to graft & Graft to surgeon \\
\hline $10-15$ & Graft shuttling & \\
\hline 7 & Glenoid knot tying & \\
\hline 12 & Medial row humeral anchors limbs' suturing to the graft & \\
\hline 12 & Medial row knot tying & \\
\hline 12 & Remnant bursal coverage/rotator cuff repair & \\
\hline 10 & Lateral row anchoring & \\
\hline 15 & Donor site irrigation and closure & \\
\hline Total time: $135-150$ & & \\
\hline
\end{tabular}




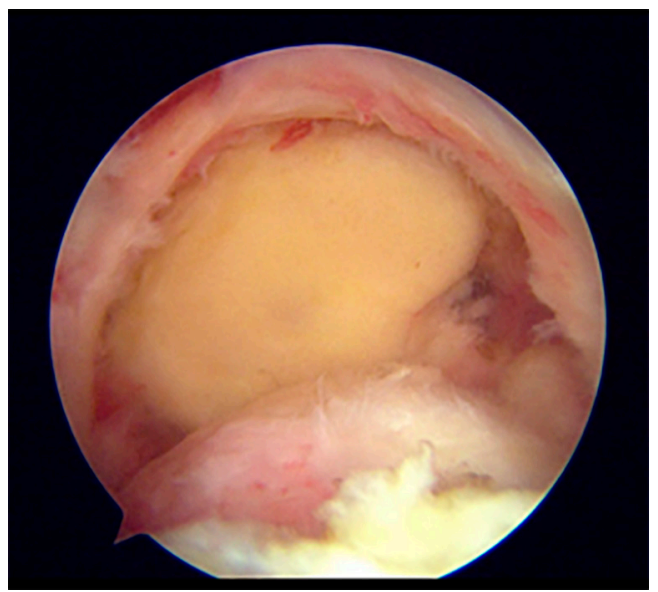

Figure 2 Diagnostic arthroscopy of a right shoulder (beach chair position) viewing from a direct lateral portal showing a massive irreparable rotator cuff tear after adequate release.

standard diagnostic arthroscopy was performed to confirm the status of the articular cartilage and the subscapularis integrity. The subscapularis integrity is important as the presence of it will associated with the clinical outcomes and complication rates following SCR (47). More importantly, detailed assessment of the rotator cuff tears should be performed after meticulous removal of the degenerative subacromial bursal tissue and articular release (Figure 2). To assess the repairability of the tendon, a retriever was usually used to grasp the tendon edge and reduce it to the footprint through an anterolateral portal. The distance between the expected anterior glenoid anchor and the expected anterior medial row humeral anchor was measured using a probe (mediolateral graft length) (23). The distance between the expected anterior to the posterior glenoid and medial humeral anchor was also measured (anteroposterior graft length). A radiofrequency ablation device was used to mark the location for the anchor. The graft harvesting and preparation are performed once the tear has been decided to be irreducible.

\section{Graft harvesting and preparation}

An ipsilateral fascia lata graft was harvested and prepared as a double-folded, 2-layered graft. To allow space for suture and knot tying, a 5-mm length was added to the final size of the graft. It is preferred to reinforce the graft construct by inserting a single layer of polypropylene mesh (Prolene $\mathrm{Mesh}^{\circledR}$; Ethicon Inc, NJ, USA) into the folded fascia lata graft in a sandwich fashion (Figure 3). A running stitch no. 2-0 polyester suture (Ethibond ${ }^{\circledR}$ ) was used to seal the graft margin. At least 6-mm-thick graft was obtained during the final graft preparation. The bursal side of the graft was marked to ease the intraarticular orientation of the graft position. A pair of heavy suture was added at the humeral side of the graft to facilitate the graft shuttling and tensioning. A saline-soaked gauze was used to cover the graft while waiting for shuttling. A routine local anesthetic injection was given to the donor site after a formal closure.

\section{Acromioplasty}

A standard anterolateral acromioplasty was performed to prevent postoperative graft attrition caused by the acromion undersurface. Acromioplasty was routinely performed as supported by the result of a previous biomechanical study in the premise of reducing subacromial contact area (48).

\section{Glenoid site preparation}

The glenoid side was prepared with the combination of radiofrequency ablation device, arthroscopic shaver, and burr after graft preparation. The remaining labrum should be cleared off from the glenoid surface. The long head of the biceps was usually very frayed and tenotomized. The superior margin of the glenoid was debrided to allow at least 3 suture anchors for fixation (JuggerKnot ${ }^{\circledR}, 2.5 \mathrm{~mm}$; Zimmer Biomet, IN, USA or Suturefix Ultra anchor ${ }^{\circledR}, 1.9 \mathrm{~mm}$; Smith \& Nephew, MA, USA) at the 10, 12, and 2 o'clock positions (Figure 4). Neviaser or accessory portal may be used to assist with the glenoid anchor placement. Care should be taken not to injure the cartilage enface of the glenoid. All soft suture anchors with smaller dimensions were preferred with the argument to avoid suprascapular nerve injury.

\section{Graft shuttling and fixation}

After the glenoid anchoring, the next step is perhaps the most challenging step in performing SCR, namely, graft shuttling. The lateral portal was extended to $2-2.5 \mathrm{~cm}$ to allow for graft shuttling with a push and pull maneuver under direct arthroscopic guidance. At this point, the posterior portal serves as the viewing portal. All sutures from the glenoid anchors were retrieved to the main working portal (direct lateral portal). Using a free needle, the graft was sutured with the glenoid anchor suture limbs externally. 

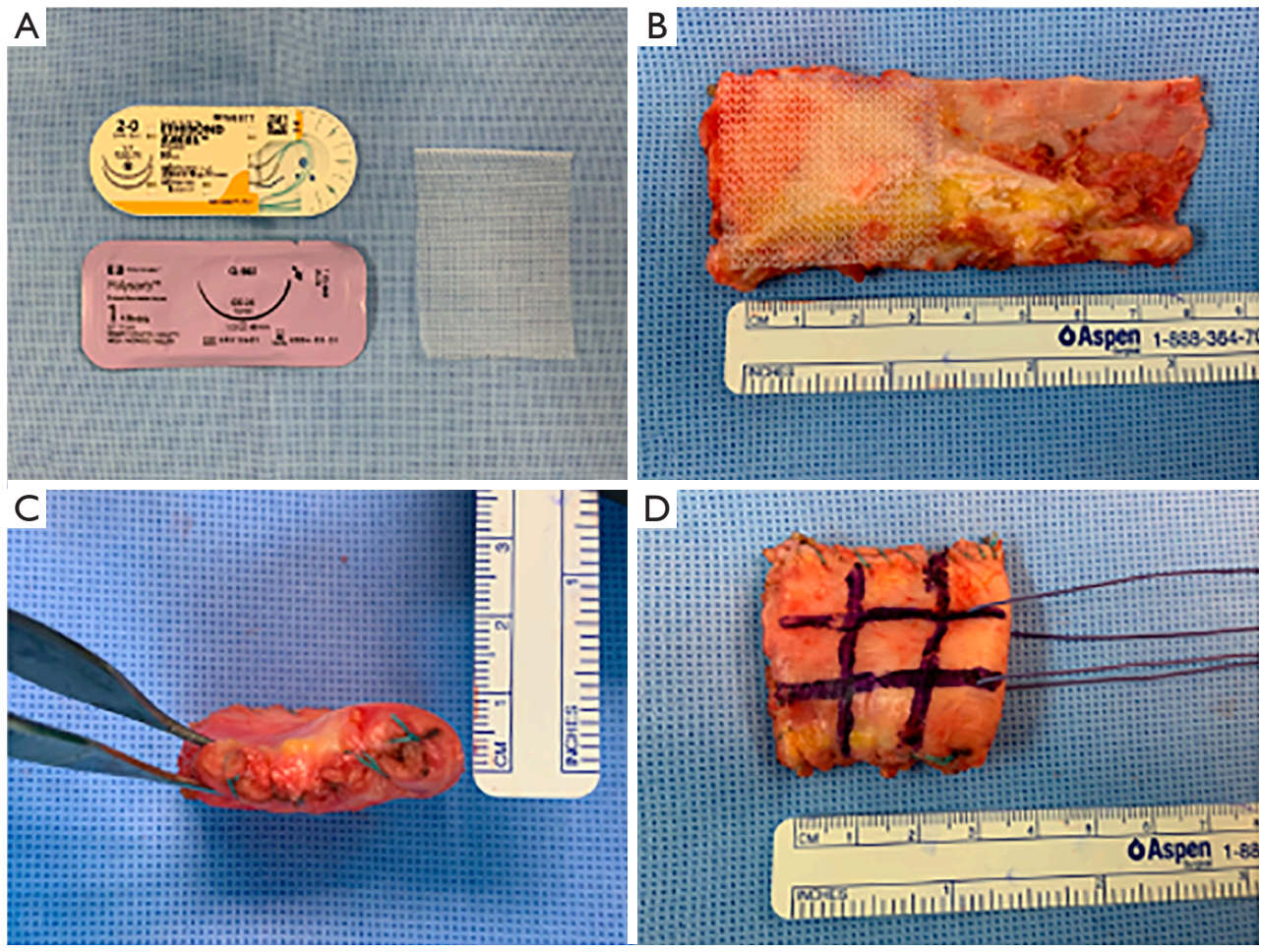

Figure 3 Graft preparation in ASCR with mesh augmentation showing 1 additional layer of polypropylene mesh (A and B) being fashioned inside the folded fascia lata (C and D).

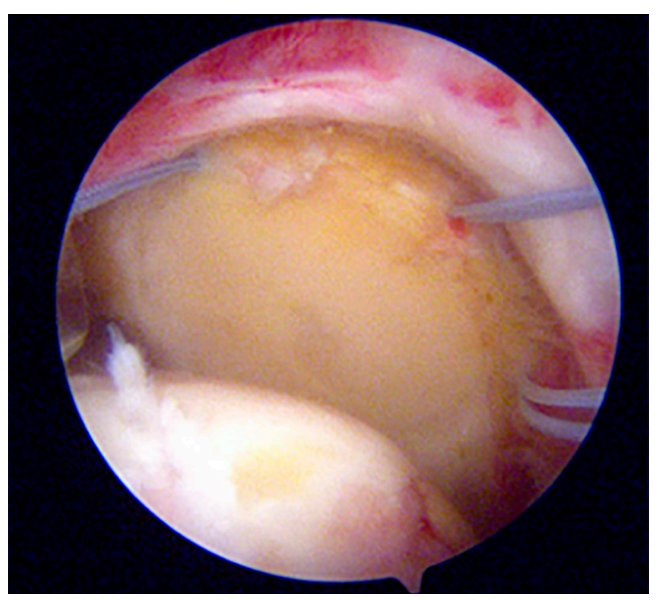

Figure 4 Three glenoid all suture anchors at the 10, 12, and 2 o'clock positions.

We do not advocate the use of an antegrade suture passer for suturing because of the risk of a broken needle tip owing to graft stiffness (from mesh reinforcement) and thickness, which was supported by a previous study (49). The graft is shuttled in by pushing the graft with a Kelly clamp by the surgeon and gently pulling the glenoid anchor suture limb by the assistant under direct arthroscopic guidance. We control the orientation of the graft by making sure that the marked side (bursal side) and the glenoid suture limb are always visible. All sutures were tied when the graft was fully seated on the glenoid. A crosslinked tie between the sutures from the 12 o'clock anchor to the 10 and 2 o'clock anchors was routinely performed.

\section{Humeral-site preparation and fixation}

Two medial anchors (Healicoil ${ }^{\circledR} 4.5 \mathrm{~mm}$; Smith \& Nephew, Andover, MA, USA) were inserted at the marked position as the medial row fixation of the humeral site. The graft was sutured to the medial row anchors using a shuttle relay technique with suture passer (Spectrum ${ }^{\circledR}$, Conmed Linvatec, Largo, FL, USA) (Figure 5). The graft was tensioned and fixed (tied) at $30^{\circ}$ shoulder abduction as recommended by a previous biomechanical study (32). 


\section{Remnant bursal coverage or rotator cuff repair}

After the medial row knot tying at the humeral site, the remaining sutures of each limb of the medial row anchors were not discarded. The remaining bursal tissue was on top of the fascia lata graft ("over-the-top") as a biological augmentation (Figure 6) (50). This was also recommended by a previous study to increase the mechanical strength of the construct $(35,51)$. This is the main concern of the author because a previous work showed that the most common graft failure is the humeral site because of the shear forces between the humerus and the undersurface of the acromion $(15,50)$. The SCR construct was completed

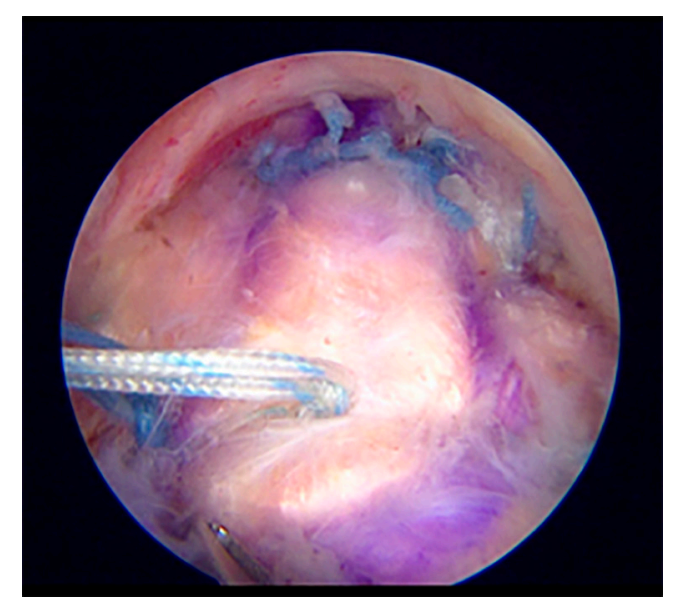

Figure $\mathbf{5}$ The graft fixed at both the glenoid and greater tuberosity sites. with the fixation of the knotless suture limbs attached to the lateral row using 2 knotless anchors (Footprint Ultra ${ }^{\circledR}$ $4.5 \mathrm{~mm}$; Smith \& Nephew, Andover, MA, USA).

\section{Postoperative rehabilitation and protocol}

In principle, the rehabilitation of a SCR is similar with the rehabilitation after MIRCT or large IRCT. After the surgery, all patients were placed in a shoulder abduction brace (in $30^{\circ}$ to $45^{\circ}$ abduction) for 6 weeks and started performing pendulum exercises for 3 weeks. After gaining range of motion after surgery, strengthening exercises for the periscapular muscles and rotator cuff were taught by a dedicated physiotherapist at 3 months after surgery. To date, there has been no studies compared the postoperative protocols after SCR. Recent systematic review showed that most of the current studies will have 3 phases of rehabilitation which consist of sling immobilization phase which ranged from 0 to 6 weeks postoperative, the active range of motion phase which ranged from 3 to 12 weeks postoperative and the strengthening phase which ranged from 6 weeks to 6 months postoperative (52). Previous studies describe that the risk of retear following large size rotator cuff repair is higher when early mobilization $(53,54)$. For this reason, delayed mobilization can be plausibly suggested after SCR rather than early mobilization to decrease graft failure rate. In our institution, a routine shoulder plain radiograph and MRI scans at 3, 6, and 12 months postoperatively were performed in all patients. MRI was preferred over ultrasonography for its accuracy in assessing graft integrity (55) (Figure 7).

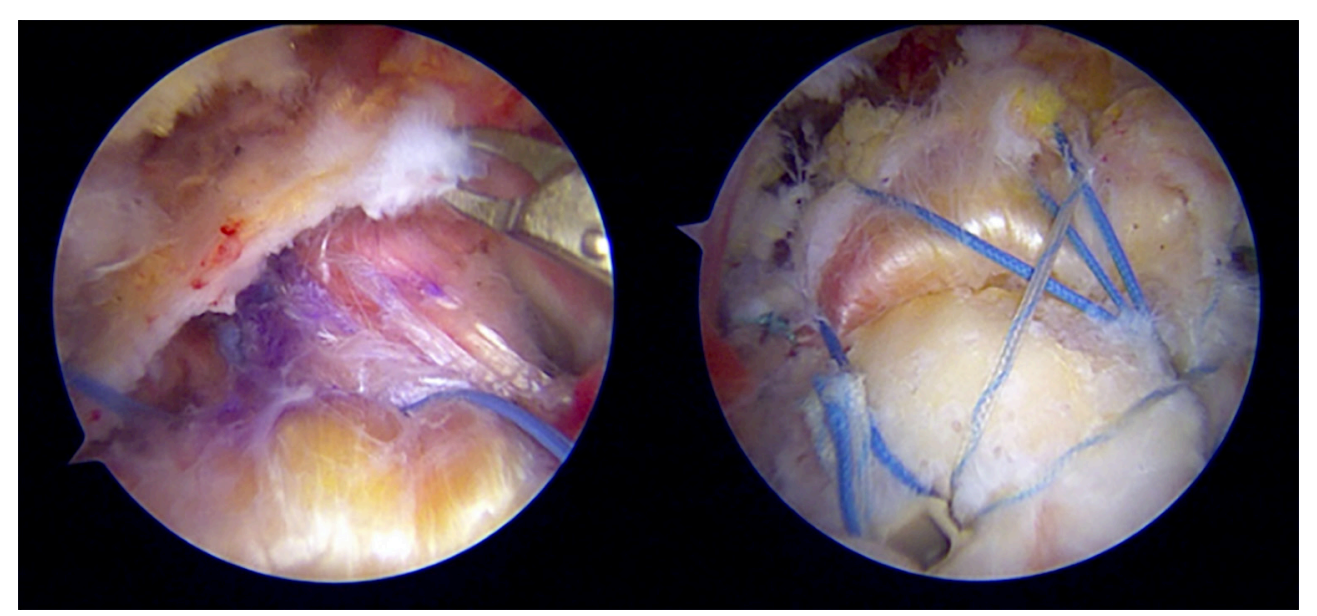

Figure 6 SCR procedure showing reconstructed capsule was superimposed by the remnant bursal tissue (50). SCR, superior capsular reconstruction. 

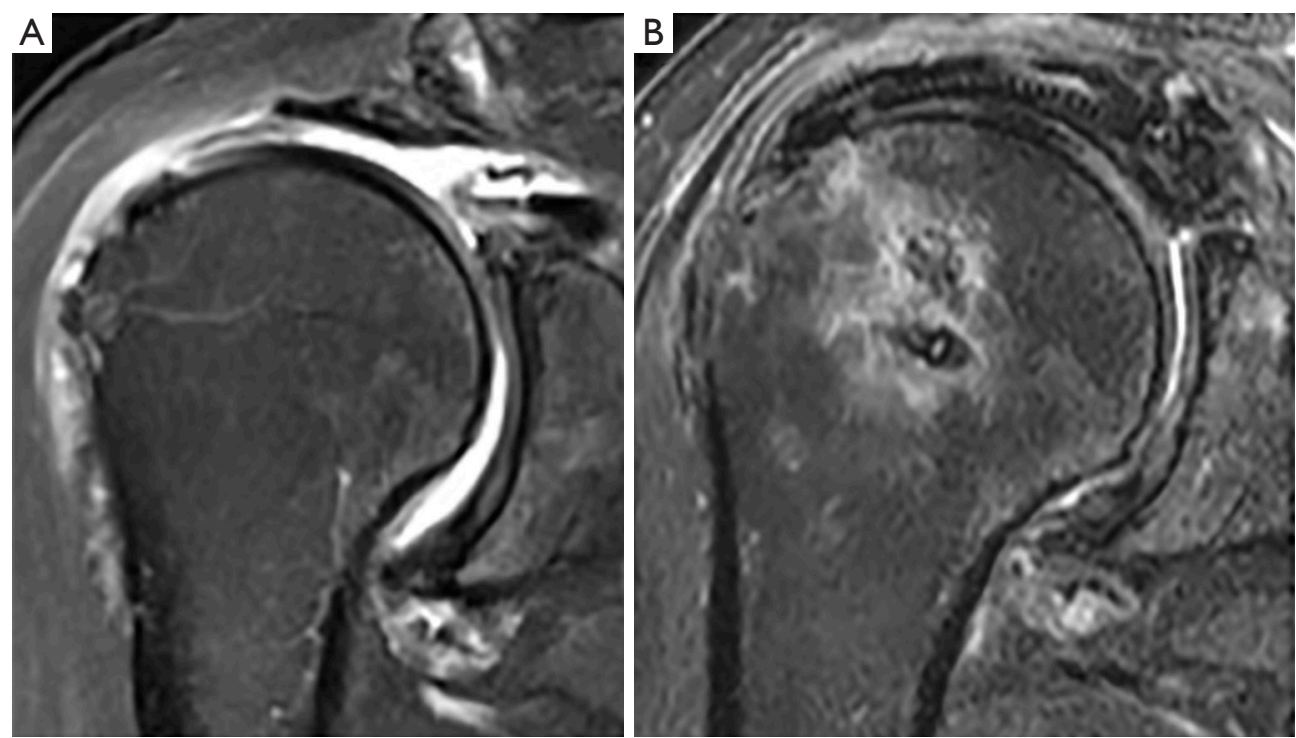

Figure 7 Preoperative T2-weighted MRI in coronal plane of a massive rotator cuff tear (A) and 12 months postoperatively (B) showing an intact graft.

\section{Discussion}

\section{Surgical outcome}

The functional outcome and graft tear rate after ASCR are variably reported owing to the differences in patient characteristics, surgical technique, and the definition of healing failure, which were limitedly reported (15). Perhaps the most compelling outcome for most surgeons was the graft failure rate that corresponds to the functional outcome and the extent that the surgical technique, particularly graft type, will affect the functional outcome.

Kholinne et al. (50) reported that the overall graft tear pattern was found at the medial row of humeral site (57.8\%), which resembles type 2 failure as described by a previous report $(15,19)$. Mihata et al. (23) reported $92 \pm 11$ points American Shoulder and Elbow Surgeons (ASES) score and graft tear rate of $16.7 \%$ at a mean follow-up of 34 months for 24 shoulders. De Campos Azevedo et al. (13) reported that postoperative simple shoulder test, subjective shoulder value, and constant score were $8.6 \pm 3.5,70 \% \pm 23 \%$, and $64 \pm 18$, respectively, and graft tear rate of $9 \%$ at a 2 -year followup for 22 shoulders. Lim et al. (15) reported $73 \pm 10$ points ASES score and graft tear rate of $29 \%$ at a mean follow-up of

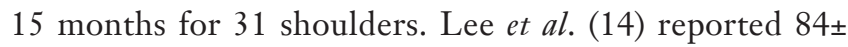
5 points ASES score and graft tear rate of $36 \%$ at a mean follow-up of 24.8 months for 32 patients. However, this study used dermal allografts and fascia lata autografts with no descriptions on allocation. Furthermore, the humeral-site graft fixation used single-row technique that may influence the failure rate, which occurred mostly at the humeral site. Dermal allograft has also been used as the graft source in several studies (19-21). Hirahara et al. (20) reported 8 patients with $86 \pm 12$ points ASES score and graft tear rate of $25 \%$ at a minimum follow-up of 2 years. Denard et al. (19) reported 59 patients with $77 \pm 22$ points ASES score and graft tear rate of $55 \%$ at the mean follow-up of 17.7 months. However, the results of graft integrity from 2 studies mentioned earlier may be underestimated because only $62.5 \%$ and $33 \%$ of patients underwent MRI confirmation, respectively. Pennington et al. (21) in a short-term study of 88 patients reported $81 \pm 10$ points ASES score and graft tear rate as 3\%. Despite the low graft tear rate, the study included younger patients (mean, 59.4; range, 27-79 years).

The summary of results of SCR from the previous significant literatures is presented in Table 4 (reproduced with permission) (56). Graft integrity was similarly reported despite the type of graft used in the surgical technique (Table 5) (56). The failure rate of the graft was $10 \%$ and $12.9 \%$ for autograft and allograft, respectively. Perhaps the most interesting thing was the reoperation rate, which was higher in the cases that used allograft $(8.2 \%)$ than those that used autograft $(3.1 \%)$. Of all 32 patients who had reoperations, the common reoperations were conversion to reverse shoulder arthroplasty (10 patients from allograft) 


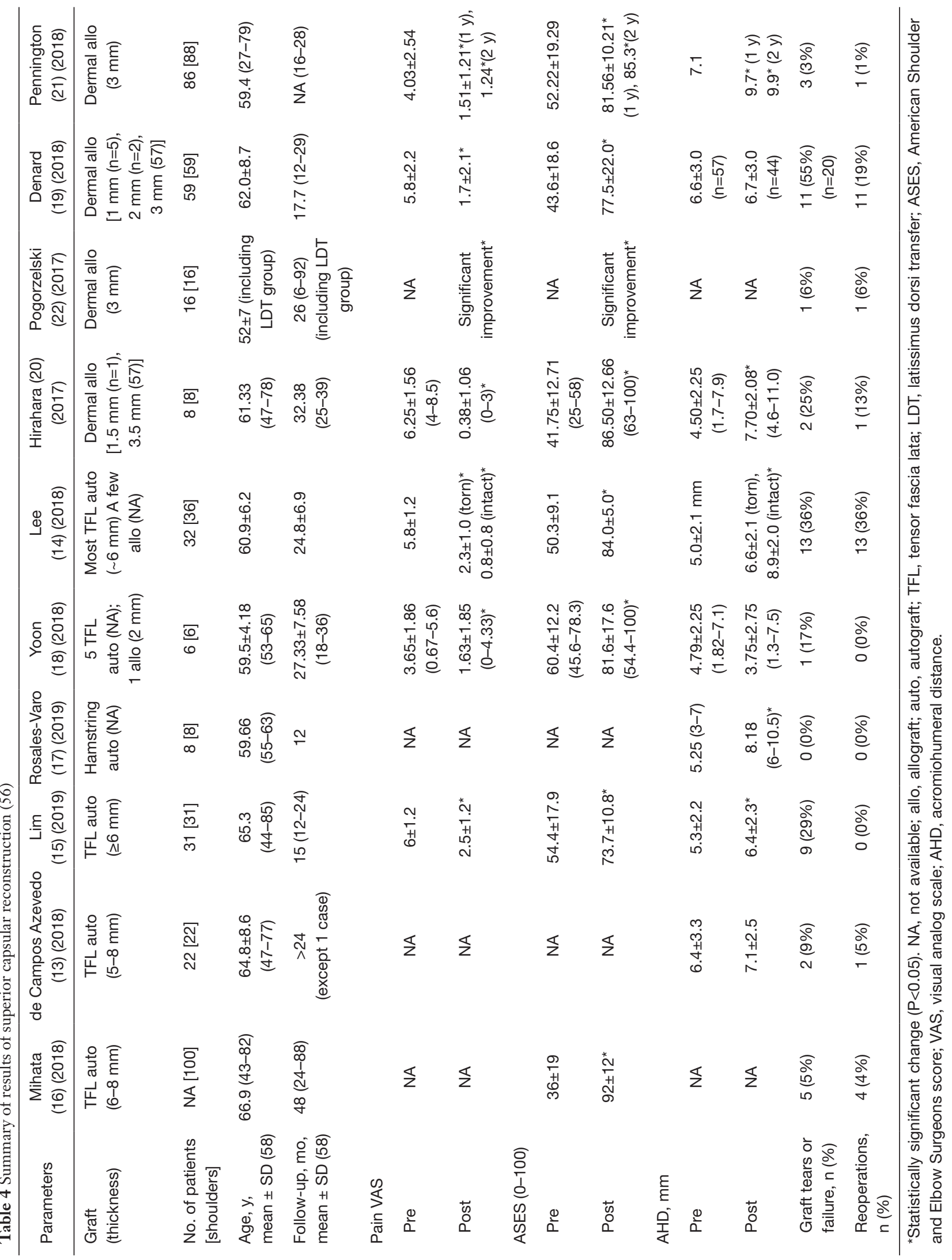


Table 5 Comparisons of graft integrity and reoperations rate between autograft and allograft in SCR (56)

\begin{tabular}{|c|c|c|c|}
\hline Parameters & Autograft & Allograft & Overall \\
\hline Number of cases/articles & $160 / 4$ & $132 / 4$ & $334 / 10$ \\
\hline Total count (\%) & $16(10.0 \%)$ & $17(12.9 \%)$ & $47(14.1 \%)$ \\
\hline \multirow[t]{5}{*}{ Details } & 7: medial row & 10: humeral site & 21: humeral suture side \\
\hline & \multirow[t]{4}{*}{$7: \mathrm{N}$} & 2: glenoid side & 4: midsubstance tear \\
\hline & & \multirow[t]{3}{*}{$1: N$} & 7: medial row \\
\hline & & & 2: lateral row \\
\hline & & & 9: $N$ \\
\hline Total count $(\%)$ & $5(3.1 \%)$ & $14(8.2 \%)$ & $32(8.6 \%)$ \\
\hline \multirow[t]{6}{*}{ Details } & $\begin{array}{l}\text { 3: arthroscopic debridement and } \\
\text { lavage because of infection }\end{array}$ & 10: rTSA & 10: rTSA \\
\hline & \multirow{5}{*}{$\begin{array}{l}\text { 2: arthroscopic capsular release } \\
\text { because of severe contracture }\end{array}$} & 1: incision and drainage & 1: incision and drainage \\
\hline & & 1: open tenodesis of biceps & 3: arthroscopic debridement and lavage \\
\hline & & \multirow[t]{3}{*}{ 2: revision SCR } & 2: arthroscopic capsular release \\
\hline & & & 1: open tenodesis of biceps \\
\hline & & & 2: revision SCR \\
\hline
\end{tabular}

rTSA, reverse total shoulder arthroplasty; SCR, superior capsular reconstruction; N, not recorded.

and revision surgery ( 2 patients from autograft and 1 patient from allograft). Majority of the published articles were with small patient numbers when outcomes were reported and being retrospectively conducted in nature. A recent systematic review showed that SCR resulted in significant improvement in patient-reported outcome measures and range of motion with the mean follow-up of the included studies were ranging from 6 to 48 months (52). However, as there were various surgical technique modifications performed in the SCR, there were not studies comparing the outcome based on the surgical technique. Up to date, the longest follow up study was reported by Mihata et al. who described arthroscopic SCR using fascia lata autograft with 5 year follow-up (59). In this mid-term follow up, the study describe that SCR provide improvement of the functional score and ROM with a low rate of graft failure rate $(10 \%)$ and high rates of return to activity (recreational sport and work).
SCR is a promising procedure for treating irreparable rotator cuff tears patients. The acceptance of superior capsular reconstruction has made it rapidly evolving in terms of a wider variety of procedures and broader surgical indications. Minimizing the graft failure rate is important for achieving good clinical outcomes following SCR. Despite the favorable and consistent outcomes reported by many researchers in the field, studies still report that outcomes are variable and largely depend on graft integrity. Future research comparing the type of graft used in SCR with a long term follow up may add a valuable contribution to the knowledge of the surgical outcome of SCR.

\section{Summary}

The management of patients with IRCTs still remains challenging despite the effort to treat with SCR. The mainstay of the challenge is imminent when patients 
are thought to be too young for the prosthetic joint replacement. Reports of studies after SCR are increasing, which mostly described encouraging results. However, with respect to functional restoration of the shoulder joint as the endpoint of the procedure, we hope to see long-term outcomes of this procedure.

\section{Acknowledgments}

Funding: None.

\section{Footnote}

Reporting Checklist: The authors have completed the Narrative Review reporting checklist. Available at http:// dx.doi.org/10.21037/atm-20-5925

Conflicts of Interest: Both authors have completed the ICMJE uniform disclosure form (available at http://dx.doi. org/10.21037/atm-20-5925). The authors have no conflicts of interest to declare.

Ethical Statement: The authors are accountable for all aspects of the work in ensuring that questions related to the accuracy or integrity of any part of the work are appropriately investigated and resolved.

Open Access Statement: This is an Open Access article distributed in accordance with the Creative Commons Attribution-NonCommercial-NoDerivs 4.0 International License (CC BY-NC-ND 4.0), which permits the noncommercial replication and distribution of the article with the strict proviso that no changes or edits are made and the original work is properly cited (including links to both the formal publication through the relevant DOI and the license). See: https://creativecommons.org/licenses/by-nc-nd/4.0/.

\section{References}

1. Rashid MS, Cooper C, Cook J, et al. Increasing age and tear size reduce rotator cuff repair healing rate at 1 year. Acta Orthop 2017;88:606-11.

2. Henry P, Wasserstein D, Park S, et al. Arthroscopic Repair for Chronic Massive Rotator Cuff Tears: A Systematic Review. Arthroscopy 2015;31:2472-80.

3. Galatz LM, Ball CM, Teefey SA, et al. The outcome and repair integrity of completely arthroscopically repaired large and massive rotator cuff tears. J Bone Joint Surg Am
2004;86:219-24.

4. Walch G, Edwards TB, Boulahia A, et al. Arthroscopic tenotomy of the long head of the biceps in the treatment of rotator cuff tears: clinical and radiographic results of 307 cases. J Shoulder Elbow Surg 2005;14:238-46.

5. Park JG, Cho NS, Song JH, et al. Long-term outcome of tuberoplasty for irreparable massive rotator cuff tears: is tuberoplasty really applicable? J Shoulder Elbow Surg 2016;25:224-31.

6. Duralde XA, Bair B. Massive rotator cuff tears: the result of partial rotator cuff repair. J Shoulder Elbow Surg 2005;14:121-7.

7. Gerber C. Latissimus dorsi transfer for the treatment of irreparable tears of the rotator cuff. Clin Orthop Relat Res 1992:152-60.

8. Bond JL, Dopirak RM, Higgins J, et al. Arthroscopic replacement of massive, irreparable rotator cuff tears using a GraftJacket allograft: technique and preliminary results. Arthroscopy 2008;24:403-9.e1.

9. Omid R, Stone MA, Lin CC, et al. Biomechanical analysis of latissimus dorsi tendon transfer with and without superior capsule reconstruction using dermal allograft. J Shoulder Elbow Surg 2019;28:1523-30.

10. Mihata T, Lee TQ, Hasegawa A, et al. Arthroscopic Superior Capsule Reconstruction Can Eliminate Pseudoparalysis in Patients With Irreparable Rotator Cuff Tears. Am J Sports Med 2018;46:2707-16.

11. Sershon RA, Van Thiel GS, Lin EC, et al. Clinical outcomes of reverse total shoulder arthroplasty in patients aged younger than 60 years. J Shoulder Elbow Surg 2014;23:395-400.

12. Moissonnier P, Carozzo C, Thibaut JL, et al. C8 cross transfer for the treatment of caudal brachial plexus avulsion in three dogs. Vet Surg 2017;46:136-44.

13. de Campos Azevedo CI, Angelo A, Vinga S. Arthroscopic Superior Capsular Reconstruction With a Minimally Invasive Harvested Fascia Lata Autograft Produces Good Clinical Results. Orthop J Sports Med 2018;6:2325967118808242.

14. Lee SJ, Min YK. Can inadequate acromiohumeral distance improvement and poor posterior remnant tissue be the predictive factors of re-tear? Preliminary outcomes of arthroscopic superior capsular reconstruction. Knee Surg Sports Traumatol Arthrosc 2018;26:2205-13.

15. Lim S, AlRamadhan H, Kwak JM, et al. Graft tears after arthroscopic superior capsule reconstruction (ASCR): pattern of failure and its correlation with clinical outcome. Arch Orthop Trauma Surg 2019;139:231-9. 
16. Mihata T, Lee TQ, Fukunishi K, et al. Return to Sports and Physical Work After Arthroscopic Superior Capsule Reconstruction Among Patients With Irreparable Rotator Cuff Tears. Am J Sports Med 2018;46:1077-83.

17. Rosales-Varo AP, Zafra M, Garcia-Espona MA, et al. Superior capsular reconstruction of irreparable rotator cuff tear using autologous hamstring graft. Rev Esp Cir Ortop Traumatol 2019;63:1-6.

18. Yoon JY, Kim PS, Jo CH. Clinical and Radiological Results after Arthroscopic Superior Capsular Reconstruction in Patients with Massive Irreparable Rotator Cuff Tears. Clinics in Shoulder and Elbow 2018;21:59-66.

19. Denard PJ, Brady PC, Adams CR, et al. Preliminary Results of Arthroscopic Superior Capsule Reconstruction with Dermal Allograft. Arthroscopy 2018;34:93-9.

20. Hirahara AM, Andersen WJ, Panero AJ. Superior Capsular Reconstruction: Clinical Outcomes After Minimum 2-Year Follow-Up. Am J Orthop (Belle Mead NJ) 2017;46:266-78.

21. Pennington WT, Bartz BA, Pauli JM, et al. Arthroscopic Superior Capsular Reconstruction With Acellular Dermal Allograft for the Treatment of Massive Irreparable Rotator Cuff Tears: Short-Term Clinical Outcomes and the Radiographic Parameter of Superior Capsular Distance. Arthroscopy 2018;34:1764-73.

22. Pogorzelski J, Horan MP, Fritz EM, et al. Arthroscopic superior capsule reconstruction (ASCR) vs Latissimus Dorsi Transfer (LDT): A comparison of early clinical outcomes. Orthop J Sports Med 2017;5:2325967117S00372.

23. Mihata T, Lee TQ, Watanabe C, et al. Clinical results of arthroscopic superior capsule reconstruction for irreparable rotator cuff tears. Arthroscopy 2013;29:459-70.

24. Ishihara $\mathrm{Y}$, Mihata T, Tamboli M, et al. Role of the superior shoulder capsule in passive stability of the glenohumeral joint. J Shoulder Elbow Surg 2014;23:642-8.

25. Burkhart SS. Fluoroscopic comparison of kinematic patterns in massive rotator cuff tears. A suspension bridge model. Clin Orthop Relat Res 1992:144-52.

26. Adams CR, DeMartino AM, Rego G, et al. The Rotator Cuff and the Superior Capsule: Why We Need Both. Arthroscopy 2016;32:2628-37.

27. Clark J, Sidles JA, Matsen FA. The relationship of the glenohumeral joint capsule to the rotator cuff. Clin Orthop Relat Res 1990;(254):29-34.

28. Vosloo M, Keough N, De Beer MA. The clinical anatomy of the insertion of the rotator cuff tendons. Eur J Orthop Surg Traumatol 2017;27:359-66.

29. Mihata T, Lee TQ, Hasegawa A, et al. Arthroscopic superior capsule reconstruction eliminates pseudoparalysis in patients with irreparable rotator cuff tears. Am J Sports Med 2018;46:2707-16.

30. Mihata T, Lee TQ, Itami Y, et al. Arthroscopic superior capsule reconstruction for irreparable rotator cuff tears: A prospective clinical study in 100 consecutive patients with 1 to 8 years of follow-up. Orthop J Sports Med 2016;4:2325967116S00076.

31. Mihata T, McGarry MH, Kahn T, et al. Biomechanical Role of Capsular Continuity in Superior Capsule Reconstruction for Irreparable Tears of the Supraspinatus Tendon. Am J Sports Med 2016;44:1423-30.

32. Mihata T, McGarry MH, Kahn T, et al. Biomechanical Effect of Thickness and Tension of Fascia Lata Graft on Glenohumeral Stability for Superior Capsule Reconstruction in Irreparable Supraspinatus Tears. Arthroscopy 2016;32:418-26.

33. Hamada K, Fukuda H, Mikasa M, et al. Roentgenographic findings in massive rotator cuff tears. A long-term observation. Clin Orthop Relat Res 1990;(254):92-6.

34. Frank RM, Cvetanovich G, Savin D, et al. Superior Capsular Reconstruction: Indications, Techniques, and Clinical Outcomes. JBJS Rev 2018;6:e10.

35. Hartzler RU, Burkhart SS. Superior Capsular Reconstruction. Orthopedics 2017;40:271-80.

36. Goutallier D, Postel JM, Bernageau J, et al. Fatty muscle degeneration in cuff ruptures. Pre- and postoperative evaluation by CT scan. Clin Orthop Relat Res 1994;(304):78-83.

37. Denard PJ, Koo SS, Murena L, et al. Pseudoparalysis: the importance of rotator cable integrity. Orthopedics 2012;35:e1353-7.

38. Burkhart SS, Hartzler RU. Superior Capsular Reconstruction Reverses Profound Pseudoparalysis in Patients With Irreparable Rotator Cuff Tears and Minimal or No Glenohumeral Arthritis. Arthroscopy 2019;35:22-8.

39. Mihata T. Editorial Commentary: Superior Capsule Reconstruction: Graft Healing for Success. Arthroscopy 2018;34:100-1.

40. Denard PJ, Brady PC, Adams CR, et al. Preliminary Results of Arthroscopic Superior Capsule Reconstruction with Dermal Allograft. Arthroscopy 2018;34:93-9.

41. Werner CM, Conrad SJ, Meyer DC, et al. Intermethod agreement and interobserver correlation of radiologic acromiohumeral distance measurements. J Shoulder Elbow Surg 2008;17:237-40.

42. Fuchs B, Weishaupt D, Zanetti M, et al. Fatty degeneration of the muscles of the rotator cuff: assessment 
by computed tomography versus magnetic resonance imaging. J Shoulder Elbow Surg 1999;8:599-605.

43. Burkhart SS, Barth JR, Richards DP, et al. Arthroscopic repair of massive rotator cuff tears with stage 3 and 4 fatty degeneration. Arthroscopy 2007;23:347-54.

44. Woodmass JM, Wagner ER, Borque KA, et al. Superior capsule reconstruction using dermal allograft: early outcomes and survival. J Shoulder Elbow Surg 2019;28:S100-9.

45. Russell RD, Knight JR, Mulligan E, et al. Structural integrity after rotator cuff repair does not correlate with patient function and pain: a meta-analysis. J Bone Joint Surg Am 2014;96:265-71.

46. Slabaugh MA, Nho SJ, Grumet RC, et al. Does the literature confirm superior clinical results in radiographically healed rotator cuffs after rotator cuff repair? Arthroscopy 2010;26:393-403.

47. Mihata T, Lee TQ, Hasegawa A, et al. Arthroscopic Superior Capsule Reconstruction for Irreparable Rotator Cuff Tears: Comparison of Clinical Outcomes With and Without Subscapularis Tear. Am J Sports Med 2020;48:3429-38.

48. Mihata T, McGarry MH, Kahn T, et al. Biomechanical Effects of Acromioplasty on Superior Capsule Reconstruction for Irreparable Supraspinatus Tendon Tears. Am J Sports Med 2016;44:191-7.

49. Kim MS, Kim DW, Choi YE, et al. Performance of antegrade suture passers according to tendon thickness. Int J Shoulder Surg 2015;9:47-51.

50. Kholinne E, Kwak JM, Kim H, et al. Arthroscopic Superior Capsular Reconstruction With Mesh Augmentation for the Treatment of Irreparable Rotator Cuff Tears: A Comparative Study of Surgical Outcomes. Am J Sports Med 2020;48:3328-38.

51. Cabarcas BC, Garcia GH, Gowd AK, et al. Arthroscopic

Cite this article as: Kholinne E, Jeon IH. Arthroscopic superior capsular reconstruction of the shoulder: a narrative review. Ann Transl Med 2021;9(3):268. doi: 10.21037/atm-205925
Superior Capsular Reconstruction and Over-the-Top Rotator Cuff Repair Incorporation for Treatment of Massive Rotator Cuff Tears. Arthrosc Tech 2018;7:e829-37.

52. Gao I, Sochacki KR, Freehill MT, et al. Superior Capsular Reconstruction: A Systematic Review of Surgical Techniques and Clinical Outcomes. Arthroscopy 2020. [Epub ahead of print]. doi: 10.1016/j.arthro.2020.09.016.

53. Bakti N, Antonios T, Phadke A, et al. Early versus delayed mobilization following rotator cuff repair. J Clin Orthop Trauma 2019;10:257-60.

54. Li S, Sun H, Luo X, et al. The clinical effect of rehabilitation following arthroscopic rotator cuff repair: A meta-analysis of early versus delayed passive motion. Medicine (Baltimore) 2018;97:e9625.

55. Badman BL, Baessler AM, Moor M. Short-Term Clinical Outcomes and Comparison of Ultrasound Versus Magnetic Resonance Imaging of Superior Capsular Reconstruction. Arthrosc Sports Med Rehabil 2020;2:e229-35.

56. Kim DM, Shin MJ, Kim H, et al. Comparison Between Autografts and Allografts in Superior Capsular Reconstruction: A Systematic Review of Outcomes. Orthop J Sports Med 2020;8:2325967120904937.

57. Giannicola G, Bullitta G, Rotini R, et al. Results of primary repair of distal triceps tendon ruptures in a general population: a multicentre study. Bone Joint J 2018;100b:610-6.

58. Grangeon M, Guillot A, Sancho PO, et al. Rehabilitation of the elbow extension with motor imagery in a patient with quadriplegia after tendon transfer. Arch Phys Med Rehabil 2010;91:1143-6.

59. Mihata T, Lee TQ, Hasegawa A, et al. Five-Year Followup of Arthroscopic Superior Capsule Reconstruction for Irreparable Rotator Cuff Tears. J Bone Joint Surg Am 2019;101:1921-30. 